\title{
Obtención y determinación de la calidad de colorante natural de uvilla para uso alimenticio
}

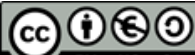

\section{Obtaining and determining the quality of uvilla natural dye for food use}

Cabrera Escobar José Omar. ${ }^{1}$, Marco Raúl Chuiza Rojas. ${ }^{2}$, Raquel Leticia Coba Carrera. ${ }^{3}$ \& Sonia Mercedes Vallejo Abarca. ${ }^{4}$

\section{Recibido: 12-04-2019 / Revisado: 28-05-209 /Aceptado: 28-06-2019/ Publicado: 25-07-2019}

\begin{abstract}
DOI: https://doi.org/10.33262/cienciadigital.v3i3.2.726

The obtaining of the grape dye is focused on the determination of its physical, chemical and microbiological characteristics, for which, we proceeded with the sensory analysis of the fruit, in which, its physical characteristics were determined, subsequently the process identification was performed The most suitable drying by means of three different dehydration equipment, determining that the best option is with the lyophilization equipment, in which, the dry product that meets the characteristics was obtained to have the best performance in the extraction of the pigment with ethanol, then the rotary evaporator was purified, recovering on the one hand and ethyl alcohol with $80 \%$ purity and on the other hand the almost pure dye, which is taken to the UV spectrophotometer to identify the structure, for which, a scanning of 400 to $600 \mathrm{~nm}$ wavelength, determining that at $427 \mathrm{~nm}$ the peak of $\beta$ Carotenes is identified, later The proximal and microbiological analysis was performed, parameters that comply with the provisions of the standard for food use. Keywords: Production, food, dye, natural, uvilla, drying, lyophilization, extraction

\footnotetext{
Escuela Superior Politécnica de Chimborazo, Facultad de Ciencias Pecuarias. Riobamba, Ecuador. jose.cabrera@espoch.edu.ec

2 Escuela Superior Politécnica de Chimborazo, Facultad de Ciencias. Riobamba, Ecuador. raulmarch_07@hotmail.com

3 Escuela Superior Politécnica de Chimborazo, Facultad de Ciencias. Riobamba, Ecuador. raquel.coba@espoch.edu.ec

4 Escuela Superior Politécnica de Chimborazo, Facultad de Ciencias. Riobamba, Ecuador. soniavallejoa@yahoo.com
} 


\section{Resumen}

La obtención del colorante de uvilla está enfocada en la determinación de sus características físicas, químicas y microbiológicas, para lo cual, se procedió con el análisis sensorial del fruto, en el cual, se determinó sus características físicas, posteriormente se realizó la identificación del proceso de secado más adecuado mediante tres equipos de deshidratación diferentes, determinando que la mejor opción es con el equipo por liofilización, en el cual, se obtuvo el producto seco que cumple las características para tener el mejor rendimiento en la extracción del pigmento con etanol, luego se procedió a la purificación en el rota vapor, recuperando por un lado un alcohol etílico con el $80 \%$ de pureza y por el otro el colorante casi puro, mismo que es llevado al espectrofotómetro UV para identificar la estructura, para lo cual, se efectuó un barrido de 400 a $600 \mathrm{~nm}$ de longitud de onda, determinando que a $427 \mathrm{~nm}$ se identifica el pico de $\beta$-Carotenos, posteriormente se realizó el análisis proximal y microbiológico, parámetros que cumplen con lo establecido en la norma para uso alimenticio.

Palabras claves: Elaboración, alimentos, colorante, natural, uvilla, secado, liofilización, extracción

\section{Introducción}

La industria alimenticia adiciona colorantes sintéticos (compuestos químicos) a productos de consumo masivo por su bajo costo, y porque mejoran sus propiedades organolépticas (vista, gusto) logrando así impresionar los órganos sensoriales de los consumidores, pero en estudios efectuados se determina que los productos químicos utilizados en la síntesis de colorantes también se consideran carcinógenos o mutagénicos, así como también sensibilizantes o alergénico, un ejemplo de pigmentos cancerígenos son los de tipo azoicos que se obtienen de arilaminas. Estudios efectuados han demostrado que los colorantes azoícos tienen potenciales carcinógenos de colon (Hassan \& et al, 2004), aumenta el nivel medio de hiperactividad en niños de 3 años, así como también entre 8 y 9 años. (Mccann $\&$ et al, 2007), por lo expuesto, se pretende obtener colorantes naturales (Brito \& et al, Obtención y determinación de la calidad de colorante a partir de las flores de Sangorache, 2019) que además de tener fuentes nutricionales ayudan a la salud de los seres humanos. 
La uvilla es un fruto (Fischer \& Miranda, 2012) silvestre nativo de los Andes Ecuatorianos (Choco \& Cazar, 2014), con un sabor agridulce, pero con gran cantidad de carbohidratos, provitamina $\mathrm{E}$ principalmente, así como $\beta$ - Carotenos, que son pigmentos naturales hidrosolubles de gran importancia para la industria alimenticia, por tal razón, pueden ser sustituidos como colorantes (Brito \& et al, Colorantes naturales para uso alimenticio, 2019) sintéticos ya que tienen características propiedades antioxidantes que inhiben la acción de especies nocivas para el organismo, como los radicales libres y se asocian con la prevención de enfermedades como el cáncer (Bui, et al., 2004), así como también puede purificar la sangre, reducir la albumina de los riñones, aliviar problemas en la garganta, próstata y bronquios, fortalecer el nervio óptico, limpiar las cataratas y prevenir la osteoporosis. (Calvo, 2009, p. 8).

La realización de este estudio se enfocó en primer lugar en el análisis sensorial (Bergquist \& Gertsson, 2017) de la uvilla, estableciendo sus características de tamaño, forma, color, entre otras, luego procedió a determinar el equipo de secado más adecuado para este proceso, utilizando tres equipos de deshidratación diferentes, determinando que el secado por liofilización es el mejor, el mismo que obtuvo un producto que cumple las características establecidas para obtener por extracción con solvente (etanol) el pigmento, a continuación se purificó por el método de destilación (Brito, Texto Básico de Operaciones Unitarias II, 2001) en el rota vapor y procedió al correspondiente análisis de sus características físicas, químicas, microbiológicas, así como también el espectro con una longitud de onda de $427 \mathrm{~nm}$ determinando a los $\beta$-Carotenos como componente principal.

\section{Metodología}

El desarrollo de la investigación se enfocó en la extracción del colorante natural (Brito \& et al, 2019) de la uvilla para el uso alimenticio mediante los procesos de secado, agitación, mezclado, extracción por solventes y destilado, para obtener un pigmento que cumpla con los estándares de calidad de acuerdo a la norma, mismo que tiene fuentes nutricionales y ayudan a la salud de los seres humanos.

La investigación se desarrolló con el objeto de extraer pigmento natural (Delgado-Vargas \& et al, 2004) a partir de la uvilla, para lo cual, se realizó el análisis sensorial de la fruta (tamaño, forma, aspecto, color, textura, olor), luego se procedió con el secado en tres equipos diferentes (liofilización, bandejas y atomización), con los datos obtenidos se 
procedió a valorar cuál de ellos es el más adecuado, determinando que el secado por liofilización (Brito, Texto Básico de Operaciones Unitarias III, 2001), es el más idóneo para obtener la materia prima, misma que es llevada a un equipo soxlet para la extracción del colorante con el uso del alcohol etílico al $90 \%$, la mezcla (Brito, Texto Básico de Operaciones Unitarias I, 2000) obtenida es llevada a un rota vapor para separar el pigmento líquido del etanol, para ello se trabaja con una temperatura de $45{ }^{\circ} \mathrm{C}$ y $250 \mathrm{mmHg}$, finalmente al producto obtenido se realiza los análisis de laboratorio físico-químicas (Brito, Borja, Chango, \& Vacacela, 2019), cuyos parámetros a valorar son pH, temperatura, densidad, índice de refracción y ${ }^{\circ} \mathrm{Bx}$, en relación a los metales son: zinc, arsénico y plomo, por otro lado, se analizó también la estructura en un espectrofotómetro Uv Visible, para lo cual, se efectuó un barrido con longitudes de onda de 400 a $600 \mathrm{~nm}$, identificando que se encuentra los $\beta$-Carotenos a $427 \mathrm{~nm}$, finalmente se efectuaron pruebas microbiológicas de mohos y levaduras, parámetros que se encuentran bajo la norma oficial mexicana nom-

\section{9-ssa1-1994.}

\section{Resultados y Discusión}

Tabla 1. Matriz comparativa para la obtención de colorante de uvilla

\begin{tabular}{|c|l|c|c|c|}
\hline \multirow{2}{*}{ No. } & \multicolumn{2}{|c|}{ Variables } & \multicolumn{3}{c|}{ Métodos de secado para la uvilla } \\
\cline { 3 - 5 } & & Bandeja & Liofilizador & Atomizador \\
\hline 1 & Tiempo de secado $(\mathrm{h})$ & 8 & 9 & 3,5 \\
\hline 2 & Temperatura de secado $\left({ }^{\circ} \mathrm{C}\right)$ & 60 & 55 & 120 \\
\hline 3 & Rendimiento (\%) & 15,30 & 17,87 & 2,30 \\
\hline 4 & Humedad (\%) & 75,8 & 73,78 & 97,48 \\
\hline 5 & Colorante extraído (\%) & 47,75 & 50,7 & 10,65 \\
\hline
\end{tabular}

Fuente: Walter Macas, Laboratorio de Investigación, 2018.

Las pruebas se las realizaron en tres equipos de secado determinando que el más adecuado es el de liofilización se utiliza la temperatura más baja a pesar de requerir mayor tiempo de secado.

Tabla 2. \% de Error f ( $\%$ de Humedad eliminada)

\begin{tabular}{|c|c|c|c|c|}
\hline Muestra & Equipo & $\begin{array}{c}\text { \% Humedad } \\
\text { Práctico }\end{array}$ & $\begin{array}{c}\text { \% } \\
\text { Humedad } \\
\text { Teórico }\end{array}$ & \% Error \\
\hline 1 & Secador de Bandejas & 75,801 & & 0,04 \\
\hline 2 & Secador por Liofilizador & 73,779 & \multirow{2}{*}{$78,9^{*}$} & 0,06 \\
\cline { 1 - 2 } & Secador por Atomización & 97,481 & & 0,24 \\
\hline
\end{tabular}

Fuente: Walter Macas, Laboratorio de Investigación, 2018. * Castro, et al., 2008, p. 228 
El porcentaje de error más bajo es el procedente del proceso de secado por bandejas y el más alto el de atomización, cabe señalar que a pesar de ser un valor mayor el de liofilización es el más adecuado por la temperatura menor y porque se mantienen las propiedades del producto.

Tabla 3. Análisis estadístico de la varianza a los parámetros físico químico

\begin{tabular}{|c|c|c|c|c|}
\hline No. & Análisis & Fruto & Colorante & Varianza (T) \\
\hline 1 & $\mathrm{pH}$ & 3,66 & 4,70 & $* * *$ \\
\hline 2 & $\rho(\mathrm{g} / \mathrm{mL})$ & 0,9863 & 0,9154 & $* * *$ \\
\hline 3 & ${ }^{\circ} \mathrm{Bx}$ & 14,7766 & 17,5334 & $* *$ \\
\hline 4 & $\mathrm{Nd}$ & 1,3920 & 1,3593 & $* *$ \\
\hline 5 & $\% \mathrm{ST}$ & 8,2050 & 22,0403 & $* * *$ \\
\hline
\end{tabular}

Fuente: Walter Macas, Laboratorio de Investigación, 2018.

Del análisis estadístico se desprende la siguiente información: es muy significativo en el pH con un nivel de confianza de 99,9\%; un nivel muy alto de significancia para la densidad con un grado de confianza de $99,9 \%$, es significativo en el ${ }^{\circ} \mathrm{Bx}$ y $\mathrm{nD}$ con un nivel de confianza de $95 \%$.

Tabla 4. Datos de longitud de onda y absorbancia

\begin{tabular}{|c|c|c|}
\hline Muestra & $\begin{array}{c}\text { Longitud de } \\
\text { onda } \boldsymbol{\lambda}(\mathbf{n m})\end{array}$ & Absorbancia \\
\hline 1 & 421 & 1,134 \\
\hline 2 & 427 & 1,414 \\
\hline
\end{tabular}

Fuente: Walter Macas, Laboratorio de Investigación, 2018.

Luego de realizar el barrido de 400 a $600 \mathrm{~nm}$ de longitud de onda en el espectrofotómetro UV se determina que el valor más adecuado y donde se determina la presencia de $\beta$ Carotenos es de $427 \mathrm{~nm}$ correspondiente inicialmente al proceso de secado por liofilización.

Tabla 5. Análisis microbiológico - Metales

\begin{tabular}{|c|c|c|c|}
\hline \multirow{2}{*}{ No. } & \multirow{2}{*}{ Parámetro } & \multicolumn{2}{|c|}{ Valor $(\mathrm{mg} / \mathrm{Kg})$} \\
\cline { 3 - 4 } & Arsénico & 3 & Experimental \\
\hline 1 & Plomo & 10 & $<0,01$ \\
\hline 3 & Zinc & 100 & 3,2 \\
\hline 4 & $\begin{array}{c}\text { Mohos y Levaduras } \\
\text { (UFC/mL) }\end{array}$ & 100 & Ausencia \\
\hline
\end{tabular}

Fuente: Walter Macas, Laboratorio de Investigación, 2018. 
Los análisis de los parámetros de metales se encuentran por debajo de la norma oficial mexicana nom-119-ssa1-1994, y existe ausencia de mohos y levaduras en el microbiológico.

\section{Conclusiones.}

$\checkmark$ Del análisis de los parámetros físico químicos se desprende los siguientes resultados: $\mathrm{pH}$ de 4,70; la densidad de $0,95 \mathrm{~g} / \mathrm{mL}$; $\operatorname{los}{ }^{\circ} \mathrm{Bx}$ de 10,69 ; el índice de refracción de 1,36 y los sólidos totales de 22,04\%.

$\checkmark$ El método de secado óptimo es por Liofilización.

$\checkmark$ La temperatura de secado es de $55^{\circ} \mathrm{C}$.

$\checkmark$ El rendimiento de extracción de colorante es del 50,70\%.

$\checkmark$ El colorante obtenido cumple con los parámetros de Arsénico, plomo y zinc, así como también para mohos y levaduras.

\section{Referencias bibliográficas}

Bergquist, S., \& Gertsson. (2017). Ascorbic acid,carotenoids, and visual quality of baby spinach as affected by shade netting and postharvest storage. Journal of Agricultural and Food Chemistry. Journal of Agricultural and Food Chemistry.

Brito, H., \& et al. (2019). Obtención del colorante natural del Camote (Ipomoea batatas). Ciencia Digital, 38-47.

Brito, H. (2000). Texto Básico de Operaciones Unitarias I. Riobamba, Chimborazo, Ecuador: Docucentro ESPOCH.

Brito, H. (2001). Texto Básico de Operaciones Unitarias II. Riobamba: Docucentro ESPOCH.

Brito, H. (2001). Texto Básico de Operaciones Unitarias III. Riobamba: Docucentro ESPOCH.

Brito, H., \& et al. (2019). Colorantes naturales para uso alimenticio. Ciencia Digital. doi:10.33262/cienciadigital.v3i2.4.510 
Brito, H., \& et al. (2019). Obtención y determinación de la calidad de colorante a partir de las flores de Sangorache. Ciencia Digital.

Brito, H., Borja, D., Chango, G., \& Vacacela, P. (2019). Obtaining Yacon Flour (Smallanthus sonchifolius). International Journal of Current Research and Academic Review.

Bui, A, V., Ngyen, M, H., \& Joachim, M. (2004). laboratory study on glucose concentration by osmotic distillation in hollow fibre module. JOURNAL OF FOOD ENGINEERING, 237-245.

Calvo, I. (2009). EL CULTIVO DE LA UCHUVA (Physalis peruviana). Microcuenca Plantón - Pacayas(10), 1-112.

Choco, O., \& Cazar, M. (2014). Variación del potencial antioxidante durante la maduración de uvilla y su estabilidad frente a tratamientos térmicos. Cuenca.

Delgado-Vargas, F., \& et al. (2004). Natural pigments:. Critical Reviews in Food Science and Nutrition, 173-289. Obtenido de https://www.clinicalkey.es/service/content/pdf/watermarked/1-s2.0S0140673607613063.pdf?locale=es_ES

Fischer, G., \& Miranda, D. (2012). Uchuva (Physalis peruviana L.). En Manual para el cultivo de frutales en el trópico (págs. 851-873). Bogotá: FISCHE R, G. (Ed.).

Hassan, O., \& et al. (2004). Extracts of cocoa (Theobroma cacao L.) leaves and their antioxidation potential. Food Chemistry.

Mccann, D., \& et al. (2007). A Randomised, Double-Blinded, Placebo-controlled Trial. Food additives and hyperactive behaviour in 3-year-old and, 1560-1567. Obtenido de https://www.clinicalkey.es/service/content/pdf/watermarked/1-s2.0S0140673607613063.pdf?locale=es_ES 


\section{PARA CITAR EL ARTÍCULO INDEXADO}

José Omar, C., Chuiza Rojas, M., Coba Carrera, R., \& Vallejo Abarca, S. (2019). Obtención y determinación de la calidad de colorante natural de uvilla para uso alimenticio. Ciencia Digital, 3(3.2), 193-200. https://doi.org/10.33262/cienciadigital.v3i3.2.726

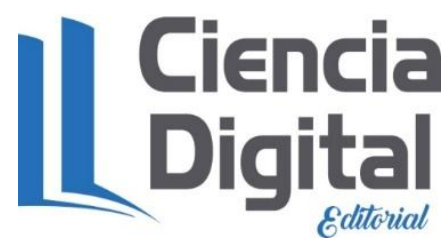

El artículo que se publica es de exclusiva responsabilidad de los autores y no necesariamente reflejan el pensamiento de la Revista Ciencia Digital.

El artículo queda en propiedad de la revista y, por tanto, su publicación parcial y/o total en otro medio tiene que ser autorizado por el director de la Revista Ciencia Digital.
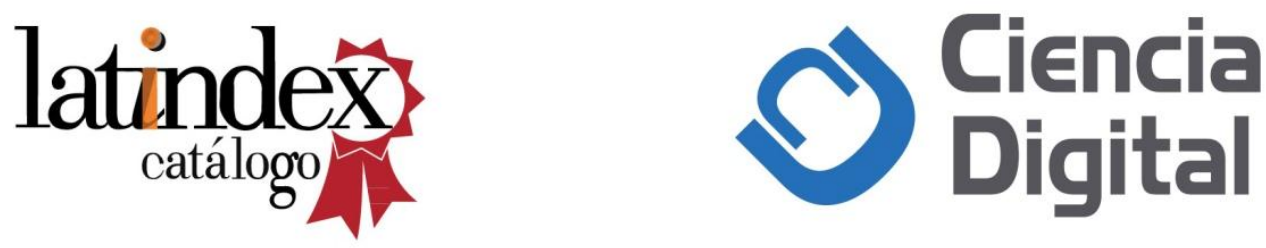